\title{
Global School-Based Student Health Survey: Country Profiles and Survey Results in the Eastern Mediterranean Region Countries
}

\section{Elham Abdalmaleki}

National Institute of Health Research (NIHR), Tehran University of Medical Sciences

Zhaleh Abdi

National Institute of Health Research (NIHR), Tehran University of Medical Sciences

Sahand Riazi Isfahani

National Institute of Health Research (NIHR)

\section{Sara Safarpoor}

National Institute of Health Research (NIHR), Tehran University of Medical Sciences

\section{Bahar Haghdoost}

National Institute of Health Research (NIHR), Tehran University of Medical Sciences

\section{Saharnaz Sazgarnejad}

National Institute of Health Research (NIHR), Tehran University of Medical Sciences

\section{Elham Ahmadnezhad ( $\nabla$ ahmadnezhad@tums.ac.ir)}

National Institute of Health Research (NIHR), Tehran University of Medical Sciences

\section{Research Article}

Keywords: Global School-based Student Health Survey, Adolescence, Eastern Mediterranean Region, Sustainable Development Goals, Universal Health Coverage, Non-communicable Disease

Posted Date: October 6th, 2021

DOl: https://doi.org/10.21203/rs.3.rs-925488/v1

License: (c) (i) This work is licensed under a Creative Commons Attribution 4.0 International License. Read Full License 


\section{Abstract}

Background: The increasing prevalence of non-communicable diseases (NCDs) has some major implications on many countries to achieve universal health coverage. This study aimed to investigate the implementation of Global School-based Student Health Survey(GSHS), which is used to assess the risk factors of NCDs among children and adolescents, by the Eastern Mediterranean Region(EMR) countries.

Methods: This study was a meta-analysis and systematic literature review of 2001-2018 published studies, which were found by searching PubMed, Google Scholar, WHO, and CDC databases. In this study, the target group was students aged between 13 and 17 years old. GSHS implementation as well as risk factors of NCDs were compared across different countries. The random-effect model for meta-analysis was considered at $95 \%$ confidence interval.

Result: In the EMR, 19 countries have implemented GSHS at least once following the survey manual (37 surveys). Overally, 201,795 young adolescents between 13 and 17 years old were included in our analysis. The overall estimation of prevalence rate of NCD risk factors among the students(13 and 15 years old), the overall estimation of the overweight prevalence rate was $24.5 \%$ (20.6-28.8), obesity prevalence rate was $7.3 \%$ (5.4-9.5), insufficient physical activity prevalence rate was $82.4 \%(80.7-84.1)$, tobacco usage prevalence rate was $14.3 \%$ (10.53-18.67), and smoking prevalence was rate $9.6 \%$ (8.111.3). In those aged from 13 to 17 years old, these rates were estimated as 19.8 (13.2-27.3), 9.7 (6.214.0), 86.1 (84.1-87.9), 17.8 (11.8-24.7), and 11.5 (9.4-13.8), respectively.

Conclusion: GSHS has been widely implemented across EMR countries. Using nationally representative data, the results show that more efforts are needed to target the NCDs risk factors among adolescents in the region.

\section{Background}

Nearly $35 \%$ of the global burden of diseases origins in adolescence, and more than 3000 adolescents die every day, mostly due to Non-communicable diseases (NCDs), injuries, and other preventable causes (1). The Eastern Mediterranean Region (EMR) suffers from some high prevalence rates of the NCD-related risk factors (2). The World Health Organization (WHO) in collaboration with other UN agencies and technical assistance of the Centers for Disease Control and Prevention (CDC) introduced Global School-based Student Health Survey (GSHS) in 2001, which has been started around the world since 2003. The purpose of GSHS is providing accurate data on health behaviors and protective factors among students, in order to help countries develop more priorities; establish appropriate programs; advocate for resources for school health programs and policies; and allow international agencies and countries to make comparisons across countries (3). The GSHS is a rapid, affordable, useful tool and a suitable alternative for surveillance systems $(4,5)$. Of note, the GSHS is a part of the WHO STEPwise approach to Surveillance (4). Correspondingly, this approach has three steps for data collection, including performing the questionnaire-based assessment, biochemical measurements, and laboratory measurements (6). In 
this regard, it is recommended to conduct GSHS every 3 to 5 years (7). This survey uses a standardized scientific sample selection process, the common school-based methodology, core questionnaire modules, and three questionnaires (including co core questionnaire modules, core-expanded questions, and country-specific questions, which are combined to form a country-specific self-administered questionnaire) (8). Accordingly, the 10 core questionnaire modules address the leading causes of morbidity and mortality, including alcohol abuse, dietary behaviors, drug abuse, hygiene, mental health status, physical activity, protective factors, sexual behaviors, tobacco usage, violence, and unintentional injury, among children and adults worldwide. Countries must select at least six of the 10 core modules in their country-specific questionnaire $(3,9)$. Thereafter, country-specific questions can be added to the GSHS core questionnaire (10). Up to now, 99 countries either have implemented this survey (11). The GSHS results could help in guiding policy makers to plan, administer, and implement some interventions targeting the NCD risk factors in adolescents (12). Following the introduction of Sustainable Development Goals (SDG) in 2015, monitoring the interventions to reduce NCD $s$ and the related risk factors globally received increasing attention $(13,14)$. Therefore, the appropriate implementation of such surveys is of great importance (15). This study aimed at examining the implementation status of GSHS in the EMR, comparing prevalence rates of NCD risk factors, including alcohol and tobacco abuse, obesity and overweight (inappropriate dietary behaviors), and physical activity among EMR countries as well as estimating an overall value for each one of these indicators in adolescents aged between 13 and 15 and between 13 and 17 years old, using the meta-analysis method.

\section{Methods}

This study was a systematic review and meta-analysis conducted in two consecutive phases. The first phase was the comparison of the surveys' designs, including questionnaires, modules, tools, and the implementation period. In addition, the second phase was the comparison of the results of these surveys, including the indicators for tobacco usage (smoking and consumption of any type of tobacco), alcohol use, obesity and overweight (inappropriate nutritional behaviors), and physical activity in terms of gender in 22 EMR countries. Afterward, the results obtained for each indicator were combined to estimate an overall value for all member states using a meta-analysis design. Prior to 2012, GSHS was only performed among students aged between 13 and 15 years old, but since 2013, the age of the target group has changed to people aged between 13 and 17 years old. Accordingly, these two age groups were compared among EMR countries. For the countries that implemented GSHS once before and once after 2013 , the results were presented for both age groups of 13-15 and 13-17 years old. However, the ages of the participants investigated in the studies performed in Iran and Saudi-Arabia were different from those of other countries in other studies. Accordingly, to preserve the consistency, their indicators were considered in the range of 13 to 17 years old.\&

\section{Phase 1- Comparison of surveys conducted across the EMR countries}


The WHO and CDC databases (survey's organizers) were considered as the main information sources for this study. In cases where there was no data from member states published on these databases, some other databases such as PubMed and Google Scholar, were searched. Keywords used for this search included: "Global School Health Survey (GSHS)", "Non-communicable disease Prevention”, "school-based survey", "Childhood and Adolescence Surveillance", "Protocol", and "methodology" along with the name of the countries with different combinations of search terms in the subjects and abstracts. Figure 1 shows PRISMA flow diagram, summarizing the review process (1). Of note, the published surveys reports were not included in the process.

The surveys were compared in terms of the modules, their sample sizes, and response rate using published country reports and factsheets between 2001 and 2018 by the WHO (11). As well, the information of Iran and Saudi-Arabia surveys were extracted from the published articles. Moreover, the questionnaires and modules were compared across the EMR countries. Finally, core modules and their frequencies of execution were compared across all these included surveys.

\section{Phase 2-comparison of the surveys' results}

The WHO dataset was used as the principal data source to examine the survey results for each country. The indicators of the Saudi-Arabia and Iran studies were extracted from both articles and national reports. The survey's results were combined using a random-effect model of meta-analysis. Thereafter, the statistical heterogeneity was examined using $\mathrm{I}^{2}$ statistic and the statistical significance level was considered as P-value $<0.05$. Due to the high heterogeneity among the studies, the random-effect model was used to combine the results at $95 \%$ confidence interval. For performing statistical analyses, the variance of each study was initially calculated by binomial distribution variance, by considering the binomial distribution of the prevalence rate. The weight given to each study was proportional to the inverse-variance. The included studies were combined in terms of the number of samples and variance. All these analyses were performed using metaprop command in STATA16 software. The cut-off points of each indicator were considered according to the definition of low, medium, and high risks (16). The definitions for each indicator as well as their cut-off points are explained in the following:

\section{Current tobacco use}

- Percentage of students who are currently smoking (smoked cigarettes on at least 1 day during the 30 days before the survey)

- Percentage of students who are currently using any tobacco products (used any tobacco products on at least 1 day during the 30 days before the survey)

- Risk Scoring: $<$ (Low); 7-15 (Medium); $\geq 16$ (High)

\section{Current alcohol use}


- Percentage of students who are currently abusing alcohol (at least one drink of alcohol for at least one day during the 30 days before the survey)

- Risk Scoring: $<20$ (Low); 20-39 (Medium); $\geq 40$ (High)

\section{Overweight or obesity.}

- Percentage of students who were overweight (>+1SD from median for BMI by age and sex)

- Percentage of students who were obese (>+2SD from median for BMI by age and sex)

- Risk Scoring: $<10$ (Low); 10-19 (Medium); $\geq 20$ (High)

\section{Physical activity.}

- Percentage of students who were physically active for at least $30 / 60$ minutes per day on $5 / 7$ days during the 7 days before the survey

\section{Physical inactivity.}

- Percentage of not engaging students who were physically active for at least 30/60 minutes per day on $5 / 7$ days during the 7 days before the survey

- Risk Scoring: $<50$ (Low); 50-69 (Medium); $\geq 70$ (High)

\section{Results}

\section{Phase 1- Comparison of the surveys}

All EMR countries, except Somalia, Iran, and Saudi-Arabia implemented at least one round of GSHS. In this region, out of $22(86 \%)$ countries, 19 ones implemented GSHS following the international protocol during 2001-2018. The target age group was defined as the students aged between 13 and 15 years old by those surveys that were implemented before 2012. The surveys conducted after 2012 defined the target age group as the students aged between 13 and 17 years old. The questionnaires used were those suggested by the international guidelines. The surveys implemented in Iran and Saudi-Arabia, which were a part of the national health surveillance system, were modified versions of the GSHS. In Iran, this modified survey, called CASPIAN study, was implemented in five rounds from 2004 to 2016 (17-25). In Saudi-Arabia, one round of Jeeluna Study was conducted in 2011-2012 (26). The age of the target group of these two studies differed from those of GSHSs implemented in other EMR countries (Saudi-Arabia: between 10 and 19 years and Iran: between 6 and18 years). No results were found for the implementation of any international or national study in Somalia. Table 1 shows the survey modules examined in EMR countries. Appendix A represents the list of available publications on GSHS for each EMR country. 
Table 1. Surveys' modules examined in EMR countries Table

\begin{tabular}{|c|l|}
\hline No & \multicolumn{1}{|c|}{ Module } \\
\hline 36 & Dietary behaviors and overweight \\
\hline 27 & Alcohol and other drug use \\
\hline 37 & Hygiene \\
\hline 32 & Physical activity \\
\hline 35 & Protective factors \\
\hline 37 & Tobacco use \\
\hline 31 & Violence and unintentional injury \\
\hline 35 & Sexual behaviours that contribute to HIV infections \\
\hline 1 & Other Sexually transmitted infections (STIs) \\
\hline 1 & Unintended pregnancies \\
\hline 1 &
\end{tabular}

Table 2 shows the included surveys' characteristics, including year(s) of implementation, sample size, and response rate percentage. The maximum sample sizes were those of Iran (21111 students in 2004) and the United Arab Emirates (15790 students in 2005). In addition, the minimum sample size belonged to Yemen in 2008 (1175 students). The highest and lowest response rates were for Jordan (99.8\%) in 2007 and Saudi-Arabia (62.25\%) in 2012, respectively.

\section{Table 2}

Characteristics of the included surveys 


\begin{tabular}{|c|c|c|c|c|}
\hline Country & $\begin{array}{l}\text { Survey's } \\
\text { Name }\end{array}$ & $\begin{array}{l}\text { Implementation } \\
\text { Year }\end{array}$ & $\begin{array}{l}\text { Sample Size } \\
\text { (Students) }\end{array}$ & $\begin{array}{l}\text { Reponses } \\
\text { Rate (\%) }\end{array}$ \\
\hline \multirow[t]{2}{*}{ Jordan } & \multirow[t]{2}{*}{ GSHS } & 2004 & 2457 & 95.0 \\
\hline & & 2007 & 2197 & 99.8 \\
\hline Afghanistan & GSHS & 2014 & 2579 & 79.0 \\
\hline \multirow[t]{5}{*}{ Iran } & \multirow[t]{5}{*}{ CASPIAN } & 2004 & 21111 & - \\
\hline & & 2007 & 9171 & - \\
\hline & & 2010 & 5570 & - \\
\hline & & 2013 & 13486 & 90.6 \\
\hline & & 2016 & 14274 & 99.0 \\
\hline \multirow[t]{3}{*}{ United Arab Emirates } & \multirow[t]{3}{*}{ GSHS } & 2005 & 15790 & 89.0 \\
\hline & & 2010 & 2581 & 91.0 \\
\hline & & 2016 & 5849 & 80.0 \\
\hline Bahrain & GSHS & 2016 & 7141 & 89.0 \\
\hline Pakistan & GSHS & 2009 & 5192 & 76.0 \\
\hline Tunisia & GSHS & 2008 & 2870 & 83.0 \\
\hline Djibouti & GSHS & 2007 & 1777 & 83.0 \\
\hline Sudan & GSHS & 2012 & 2211 & 77.0 \\
\hline Syrian Arab Republic & GSHS & 2010 & 3102 & 97.0 \\
\hline Somalia & - & - & - & - \\
\hline Iraq & GSHS & 2012 & 2038 & 88.0 \\
\hline Saudi Arabia & Jeeluna & 2012 & 12121 & 62.2 \\
\hline \multirow[t]{3}{*}{ Oman } & GSHS & 2005 & 2979 & 97.0 \\
\hline & & 2010 & 1606 & 89.0 \\
\hline & & 2015 & 3468 & 92.0 \\
\hline Qatar & GSHS & 2011 & 2021 & 87.0 \\
\hline \multirow[t]{2}{*}{ Kuwait } & GSHS & 2011 & 2672 & 85.0 \\
\hline & & 2015 & 3637 & 78.0 \\
\hline \multirow[t]{2}{*}{ Palestine Gaza } & GSHS & 2010 & 2677 & 95.0 \\
\hline & & & 2122 & 95.0 \\
\hline
\end{tabular}




\begin{tabular}{|c|c|c|c|c|c|}
\hline \multicolumn{2}{|c|}{ Gaza ${ }^{[1]}$ UNWRA } & & & \multirow[b]{2}{*}{2187} & \multirow[b]{2}{*}{96.0} \\
\hline & UNWRA & & & & \\
\hline & Lebanon & & & & \\
\hline & UNWRA & & & 2120 & 94.0 \\
\hline & Syrian & & & & \\
\hline & UNWRA & & & 2015 & 90.0 \\
\hline & West Bank & & & & \\
\hline & West Bank & & & 1908 & 94.0 \\
\hline & UNWRA & & & 1529 & 93.0 \\
\hline & Jordan & & & & \\
\hline Lebanon & & GSHS & 2005 & 5115 & 88.0 \\
\hline & & & 2011 & 2286 & 87.0 \\
\hline & & & 2017 & 5708 & 82.0 \\
\hline Libya & & GSHS & 2007 & 2242 & 98.0 \\
\hline Morocco & & GSHS & 2006 & 2670 & 84.0 \\
\hline & & & 2010 & 2924 & 92.0 \\
\hline & & & 2016 & 6745 & 91.0 \\
\hline Egypt & & GSHS & 2006 & 5249 & 87.0 \\
\hline & & & 2011 & 2568 & 85.0 \\
\hline Yemen & & GSHS & 2008 & 1175 & 82.0 \\
\hline & & & 2014 & 2655 & 75.0 \\
\hline
\end{tabular}

\section{Phase 2-Comparison of the surveys' results}

Table 3 presents the risk levels of the non-communicable diseases' risk factors.

\section{Tobacco Use}

Risk levels for tobacco usage were defined as follows: 
As shown in Table 3, male adolescents aged between 13 and 15 years old smoke cigarettes or any type of tobacco products at higher rates compared to the female ones at the same age. The highest prevalence rate of cigarette smoking in this age group among men was 25\% in Qatar (2011) followed by Palestine (24\% in 2010). The lowest prevalence rate of smoking cigarettes at this age group was reported as approximately $1 \%$ in women in Libya (2007), Morocco (2006), and Egypt (2011). The highest prevalence rates of using any type of tobacco products in this age group were seen in male adolescents living in Lebanon (2017) and Kuwait (2015), as 35\% and 29\%, respectively. As well, the lowest prevalence rate was reported as $3 \%$ among women in Tunisia (2007), Libya. (2007), and Morocco (2006).

The prevalence rate of total tobacco usage (cigarettes and any type of tobacco products) among students aged between 13 and 15 years old ranged was 18\% in Qatar (2011) and 3\% in Morocco (2003). The highest prevalence rate for smoking any type of tobacco products in this age group was $31.5 \%$ in Lebanon (2017) and the lowest one was 6\% in Libya (2007) and Morocco (2006)

\section{Physical activity}

Risk levels for physical inactivity were defined as follows:

$70 \%$ or above

$50-69 \%$

Below $50 \%$

The results shown in Table 3 indicated that the prevalence of physical activity is relatively low among adolescents in the EMR region.

\section{Inappropriate dietary behaviors (obesity and overweight)}

Risk levels for both obesity and overweight were defined as follows:

$20 \%$ or above

$10-19 \%$

Below 10\%

As shown in Table 3, the prevalence of overweight is relatively high in both female and male adolescents. The highest prevalence rates of overweight and obesity were reported in men in Kuwait (56\% and 28\%, respectively) and the lowest levels were reported in Pakistan ( $5 \%$ and $1 \%$, respectively). The overall prevalence of overweight, obesity, and physical inactivity were observed as follows: Kuwait has the highest and Pakistan has the lowest prevalence rates of overweight. The prevalence rate of obesity ranged from $1 \%$ in Pakistan to $23.6 \%$ in Kuwait. The highest prevalence of physical inactivity was reported in Egypt and Afghanistan, as $90.6 \%$ and $90.4 \%$ respectively. Of note, the lowest prevalence rate was observed in Lebanon (65.4\%). 


\section{Alcohol consumption}

Risk levels for alcohol consumption were defined as follows:

\section{$40 \%$ or above

The prevalence of alcohol consumption was reported only for three countries, including Lebanon, Morocco, and Syria. In all these three countries, alcohol consumption was higher among men.

As Table 4 shows, among the students aged between 13 and 17 years old, the highest and lowest overweight prevalence rates were reported in Kuwait (52.9\%) and Iran (4.3\%). In addition, the highest and lowest obesity prevalence rates were reported in Kuwait (28.2\%) and Yemen (1.7\%), among male adolescents, respectively. The prevalence of physical activity was relatively low among adolescents aged 13-17 years in the region. The highest and lowest physical inactivity prevalence rates in this age group were in Afghanistan (90.7\%) and Bahrain (80.5\%), respectively. The highest prevalence of cigarettes smokers and any type of tobacco products in this age group was in Kuwait (28.2\%) and Lebanon (40.9\%), among male adolescents, respectively.

In this study, 37 reports and 10 articles were selected and included to obtain an overall estimation for each indicator by the meta-analysis. The total number of the study participants was 201795 . The heterogeneity statistics for all the indicators was obtained as approximately $\mathrm{I}^{2}>98$ by combining the results and calculating the overall estimation using a random effect model. Accordingly, this indicated that the results of the studies were extremely heterogeneous. Table 5 shows the total prevalence of overweight, obesity, physical inactivity, and tobacco use (any products and cigarettes) by the two age groups in the EMR. Sex subgroup level analysis was also performed to reduce the existing heterogeneity in results and the heterogeneous results were reported to be high yet (nearly $\left.\right|^{2}>99$ ).

\section{Table 5}

\section{Result of meta-analysis and heterogeneity on prevalence of overweight, obesity, physical inactivity, tobacco use (any products \& cigarettes) by age group (13-15 \& 13-17 years old)}




\begin{tabular}{|c|c|c|c|c|c|c|}
\hline \multicolumn{7}{|c|}{ Overall (all EMR countries) } \\
\hline \multirow{2}{*}{$\begin{array}{l}\text { Age } \\
\text { group }\end{array}$} & \multirow[t]{2}{*}{ Result } & \multicolumn{2}{|c|}{ Dietary Behaviors } & \multirow{2}{*}{$\begin{array}{l}\text { Physical } \\
\text { Inactivity }\end{array}$} & \multicolumn{2}{|c|}{ Tobacco Use } \\
\hline & & overweight & obese & & $\begin{array}{l}\text { Any } \\
\text { Products }\end{array}$ & Cigarettes \\
\hline \multirow[t]{4}{*}{$13-15$} & \multirow{2}{*}{$\begin{array}{l}\text { pooled estimate } \\
\text { prevalence } \\
(95 \% \mathrm{Cl})\end{array}$} & \multirow{2}{*}{$\begin{array}{l}24.5 \\
(20.6-28.8)\end{array}$} & \multirow{2}{*}{$\begin{array}{l}7.3 \\
(5.4- \\
9.5)\end{array}$} & \multirow{2}{*}{$\begin{array}{l}82.4 \\
(80.7-84.1)\end{array}$} & \multirow{2}{*}{$\begin{array}{l}14.3 \\
(10.53- \\
18.67)\end{array}$} & \multirow{2}{*}{$\begin{array}{l}9.6 \\
(8.1-11.3)\end{array}$} \\
\hline & & & & & & \\
\hline & $1^{2}$ & 99.6 & 99.4 & 98.3 & 99.5 & 98.7 \\
\hline & Pvalue $^{*}$ & \multicolumn{2}{|l|}{$P<0.001$} & & & \\
\hline \multirow[t]{4}{*}{$13-17$} & $\begin{array}{l}\text { pooled estimate } \\
\text { prevalence }\end{array}$ & \multirow{2}{*}{$\begin{array}{l}19.8 \\
(13.2-27.3)\end{array}$} & \multirow{2}{*}{$\begin{array}{l}9.7 \\
(6.2- \\
14.0)\end{array}$} & \multirow{2}{*}{$\begin{array}{l}86.1 \\
(84.1-87.9)\end{array}$} & \multirow{2}{*}{$\begin{array}{l}17.8 \\
(11.8- \\
24.7)\end{array}$} & \multirow{2}{*}{$\begin{array}{l}11.5 \\
(9.41- \\
13.89\end{array}$} \\
\hline & $\begin{array}{l}\text { prevarence } \\
(95 \% \mathrm{Cl})\end{array}$ & & & & & \\
\hline & $1^{2}$ & 99.8 & 99.7 & 98.3 & 99.6 & 99.0 \\
\hline & P-value ${ }^{*}$ & $P<0.001$ & & & & \\
\hline
\end{tabular}

\section{Discussion}

This study aimed at examining the implementation status of GSHS in the EMR, comparing prevalence rates of the NCD risk factors, including, alcohol and tobacco use, obesity and overweight (inappropriate dietary behaviors), and physical activity among EMR countries as well as estimating an overall value for each indicator among adolescents aged between 13 and 15 and between 13 and 17 years old.

All the countries in the EMR, except Iran, Saudi-Arabia, and Somalia, have implemented the GSHS.

Therefore, GSHS has been started widely across EMR. However, the implemented surveys considerably differed in terms of sample sizes. The CASPIAN study conducted in Iran reported the highest sample size among the other implemented surveys. This review showed that EMR countries have not implemented GSHS in a harmonized manner, and this can allow the comparison of relative indicators among member states over time. Of note, in the implemented surveys, most of the internationally recommended modules were considered. However, some subjects such as high-risk sexual behaviors, alcohol and drug use, and reproductive health were not considered in the included studies, mainly due to the cultural factors. Therefore, their prevalence rates were not reported in this study. 
The results for the two age groups of between 13 and 15 and between 13 and 17 years old are separately presented in the present study. The prevalence rate of physical inactivity in both age groups was estimated to be more than $80 \%(P<0.001)$. In addition, the risk level was high in most years. The lowest prevalence rate was reported among adolescents aged between 13 and 17 years old living in Afghanistan in 2014. In all the countries, except Sudan and Afghanistan, the prevalence of physical inactivity was higher in male adolescents in comparison to that of female ones. The prevalence rate of overweight in the region in both age groups was high as well ( 24.5 and $19.8 \%$, respectively). The results indicate that countries in where students are more physically inactive, are more likely to have higher prevalence of overweight and obesity. Nowadays, more than $80 \%$ of the world's adolescent population is insufficiently physically active (27). Policies to reduce insufficient physical activity are being implemented in only $56 \%$ of WHO Member States in $2013(28,29)$. Moreover, similar to other countries in the world, the EMR countries had no considerable progress. In this regard, a reduction of at least $10 \%$ in physical inactivity is recommended up to $2025(28,30)$. Additionally, because of its high prevalence, it should be regarded as a high priority public health problem in EMR countries. Some factors that may contribute into the high prevalence of this factor in the EMR are the followings: lack of access to recreational facilities, lack of outdoor spaces (like parks), inappropriate public transportation, and social and cultural norms in the region. Women mostly face some personal and social barriers to be engaged in physical activities. Therefore, it is highly recommended to consider country-specific sociocultural and environmental factors affecting physical activity in the design of appropriate interventions. Our results indicate that the prevalence of overweight among girls due to their low physical activity is higher than boys. The highest and lowest prevalence rates of overweight and obesity were reported in Kuwait and Pakistan, respectively. In another systematic review, the physical inactivity status among Arabic-speaking countries was examined in 2018, its prevalence was reported to be very high, which is similar to our results (31). It was shown that school-based interventions can have important potentials for both obesity prevention and physical activity promotion among school-aged children and adolescents. In this regard, strategies proposed for promoting behavioral change need to take the strong role of the family supports into the account.

In the EMR region, the prevalence of tobacco us was higher in male adolescents than female ones. The highest and lowest prevalence rates were reported in Lebanon (31.5\%) and Morocco (3\%), respectively. Furthermore, the overall prevalence of tobacco usage in the region was reported as $14.3 \%$ among $13-15$ years old age group and as $17.8 \%$ among $13-17$ years old age group. Similar to the other parts of the world, rates of tobacco usage among adolescents and young people are increasing in the EMR region as well (32). Resolution EM / RC64 / 5, was adopted by the 61st session of the Regional Committee for the Eastern Mediterranean in 2017, which urged member states to implement the effective interventions on the prevention and control of the non-communicable diseases (33).

It may be difficult to achieve all SDG 3 targets in the EMR up to 2030. The high prevalence of the risk factors of NCDs among adolescents in this region demonstrated that interventions aiming at reducing risk factors associated with NCDs, have not achieved the expected results so far. It is highly recommended to include culturally relevant, evidence-based trainings in the school curricula. 
Correspondingly, this could be considered as one of the best opportunities to correct the lifestyle of this age group. As well, it is necessary to provide sufficient facilities for the purpose of improving the physical activity level of young people as well as promoting healthy eating behaviors in schools. This issue should be considered wen the allocation of the educational budgets in this region.

To prioritize the interventions needed to tackle the NCDs risk factors based on our results, physical inactivity was found as the most important modifiable risk factor that should be addressed by EMR countries in the near future. Considering the high prevalence of youth smoking in the EMR, it is suggested that tobacco usage in adolescents and its contributing factors be explored further in the region. A coalition of youth advocates for health in the EMR was launched in 2017 and the issue needs to be emphasized in future meetings of the coalition.

\section{Conclusion}

Considering the fact that the implementation of GSHS survey received considerable attentions of EMR member states, so it can be concluded that the risk factors of NCDs in the adolescents are reasonably monitored in the region. As a further step, a harmonized survey could be designed and then implemented in the region, in order to facilitate the comparison of the results across the countries. Although the NCD risk factors are being monitored through GSHS surveys, it seems that still many targets related to the risk factors have not been achieved in the region. In addition, it is critical that comprehensive policies be further designed to address adolescent health issues and to measure the impact of any intervention at both regional and country levels. Furthermore, despite the implementation of GSHS, it seems that an appropriate surveillance system should be designed across the region to monitor the current situation of the NCD risk factors among young people. However, survey's implementation per se cannot guarantee the proper monitoring of the NCD risk factors. As well, the capacity of EMR countries for surveillance of NCDs, their risk factors and determinants, as well as utilizing the obtained results in NCD programs' monitoring and evaluation should be strengthened. Among the examined risk factors in this study, insufficient physical activity in adolescents, which needs an instant action to prevent and control, should be promptly considered as a major public health problem by all countries. A comprehensive, multisectoral program to promote physical activity is urgently needed in order to reduce the economic and health burdens of physical inactivity in this region.

\section{Abbreviations}

non-communicable diseases (NCDs)

Global School-based Student Health Survey (GSHS)

Eastern Mediterranean Region (EMR)

The World Health Organization (WHO) 


\section{Declarations}

\section{- Ethics approval and consent to participate}

This is a systematic Review with no ethical code and no need for consent particate

\section{- Consent for publication}

This paper does not include the individual data

\section{- Availability of data and materials}

The datasets generated and/or analyzed during the current study are not publicly available due to they don't not provide more necessary information, but are available from the corresponding author on reasonable request.

\section{- Competing interests}

The authors declare that they have no competing interests

\section{- Funding}

This research did not receive any specific grant from funding agencies in the public, commercial, or notfor-profit sectors.

\section{- Authors' contributions}

EA, EAb, and ZhA conceived the paper. EA, SR, and EAb, conducted the statistical analysis and drafted the manuscript. EA, BH, SHS, and ZhA reviewed and edited the manuscript. SS coordinated the overall process. All authors reviewed the manuscript, provided edits, and approved the final manuscript.

\section{- Acknowledgments}

The authors would like to thank the anonymous reviewers for their insightful suggestions and careful reading of the manuscript.

\section{- Authors' information}

Elham Abdalmaleki ${ }^{1}$, Zhaleh Abdi ${ }^{2}$, Sahand Riazi Isfahani ${ }^{2}$, Sara Safarpoor ${ }^{1}$, Bahar Haghdoost ${ }^{3}$, Saharnaz Sazgarnejad ${ }^{3}$, Elham Ahmadnezhad ${ }^{4}$ 
1-MSc, Researcher, National Institute of Health Research (NIHR), Tehran University of Medical Sciences, Tehran, Iran

2- Assistant Professor, National Institute of Health Research, Tehran University of Medical Sciences, Tehran, Iran

3.MD-MPH student, School of medicine, Tehran University of Medical Sciences, Tehran, Iran researcher,National Institute of Health Research (NIHR), Tehran University of Medical Sciences, Tehran, Iran

4. Associate Professor, National Institute of Health Research, Tehran University of Medical Sciences, Tehran, Iran

Corresponding author: Elham Ahmadnezhad, ahmadnezhad@tums.ac.ir

\section{References}

1. WHO. Noncommunicable Disease Prevention and Adolescents [updated December 2017]. Available from: https://plan-uk.org/file/ncd-prevention-and-adolescents-report/download?token=tkG9kOxg.

2. Fikri $\mathrm{M}$, Hammerich A. Scaling up action on the prevention and control of noncommunicable diseases in the WHO Eastern Mediterranean Region. Eastern Mediterranean Health Journal. 2018;24(1):3-4.

3. WHO. Global School-based Student Health Survey (GSHS) 2018. Available from: https://www.who.int/teams/noncommunicable-diseases/surveillance/systems-tools/global-schoolbased-student-health-survey.

4. GHDx. STEPS Global School-Based Student Health Survey (GSHS) [updated Jan 7, 2019]. Available from: http://ghdx.healthdata.org/series/steps-global-school-based-student-health-survey-gshs.

5. Abdalmaleki E, Abdi Z, Goharimehr M, Alvandi R, Esfahani S, Ahmadnezhad E. A review of the methodology and tools of childhood \& adolescence surveillance and prevention of adult noncommunicable disease survey (CASPIAN) conducted in Iran. Iranian Journal of Epidemiology. 2019;15(3).

6. WHO. STEPwise Approach to NCD Risk Factor Surveillance (STEPS). Available from: https://www.who.int/teams/noncommunicable-diseases/surveillance/systems-tools/steps.

7. CDC. GSHS Data User's Guide 2013 [updated January 9, 2018]. Available from: https://www.cdc.gov/gshs/background/pdf/gshs-data-users-guide.pdf.

8. PAHO. Global School-based Student Health Survey (GSHS). Available from: https://www3.paho.org/hq/index.php? option=com_content\&view=article\&id=13582:gshs\&ltemid=42359\&lang=en\#\&ltemid=42360. 
9. WHO. GSHS Questionnaire. Available from: https://www.who.int/teams/noncommunicablediseases/surveillance/systems-tools/global-school-based-student-health-survey/questionnaire.

10. Page RM, Danielson M. Multi-country, cross-national comparison of youth tobacco use: findings from global school-based health surveys. Addictive behaviors. 2011;36(5):470-8.

11. WHO. Global School-based Student Health Survey (GSHS)_Data \& Reporting. Available from: https://www.who.int/teams/noncommunicable-diseases/surveillance/data.

12. Organization WH. Global school-based student health survey (GSHS) 2004 Core Questionnaire Module Rationale. 2004.

13. Abdi Z, Majdzadeh R, Ahmadnezhad E. Developing a framework for the monitoring and evaluation of the Health Transformation Plan in the Islamic Republic of Iran: lessons learned. Eastern Mediterranean Health Journal. 2019;25(6).

14. Lozano R, Fullman N, Abate D, Abay SM, Abbafati C, Abbasi N, et al. Measuring progress from 1990 to 2017 and projecting attainment to 2030 of the health-related Sustainable Development Goals for 195 countries and territories: a systematic analysis for the Global Burden of Disease Study 2017. The Lancet. 2018;392(10159):2091-138.

15. Ali Akbar Haghdoost MA, Lari, Iraj Harirchi, Elham Ahmadnezhad. Universal Health Coverage Road Map in Eastern Mediterranean Region: Brief Report on Ministerial Meeting, 03-05 September (2018), Salaleh, Oman. Hakim Health Systems research journal.21(2):147-52.

16. Naik R, Kaneda T. Addressing noncommunicable disease risk factors among young people: Asia's window of opportunity to curb a growing epidemic. Washington, DC: Population Reference Bureau. 2016.

17. Kelishadi R, Hovsepian S, Qorbani M, Jamshidi F, Fallah Z, Djalalinia S, et al. National and subnational prevalence, trend, and burden of cardiometabolic risk factors in Iranian children and adolescents, 1990-2013 (study protocol). Archives of Iranian medicine. 2014;17(1):71-80.

18. Kelishadi R, Heshmat R, Motlagh ME, Majdzadeh R, Keramatian K, Qorbani M, et al. Methodology and early findings of the third survey of CASPIAN study: A national school-based surveillance of students' high risk behaviors. International journal of preventive medicine. 2012;3(6):394.

19. Kelishadi R, Ardalan G, Qorbani M, Ataie-Jafari A, Bahreynian M, Taslimi M, et al. Methodology and early findings of the fourth survey of childhood and adolescence surveillance and prevention of adult non-communicable disease in Iran: The CASPIAN-IV study. International journal of preventive medicine. 2013;4(12):1451.

20. Motlagh ME, Ziaodini H, Qorbani M, Taheri M, Aminaei T, Goodarzi A, et al. Methodology and early findings of the fifth survey of childhood and adolescence surveillance and prevention of adult noncommunicable disease: the CASPIAN-V study. International journal of preventive medicine. $2017 ; 8$.

21. Kelishadi R, Amirkhani A, Ardalan G, ZIA AS, Majdzadeh R. AN OVERVIEW OF A NATIONAL SURVEILLANCE PROGRAM IN IRAN FOR PREVENTION OF CHRONIC NON-COMMUNICABLE DIS- 
EASES FROM CHILDHOOD: CASPIAN STUDY. Iranian Journal of Public Health. 2009(Vol 38 No Supple 1 (2009)):102-6.

22. Khashayar P, Kasaeian A, Heshmat R, Motlagh ME, Mahdavi Gorabi A, Noroozi M, et al. Childhood overweight and obesity and associated factors in iranian children and adolescents: a multilevel analysis; the CASPIAN-IV study. Frontiers in pediatrics. 2018;6:393.

23. Kelishadi R, Ardalan G, Gheiratmand R, Majdzadeh R, Hosseini M, Gouya M, et al. Thinness, overweight and obesity in a national sample of Iranian children and adolescents: CASPIAN Study. Child: care, health and development. 2008;34(1):44-54.

24. Kelishadi R, Ardalan G, Gheiratmand R, Gouya MM, Razaghi EM, Delavari A, et al. Association of physical activity and dietary behaviours in relation to the body mass index in a national sample of Iranian children and adolescents: CASPIAN Study. Bulletin of the World Health Organization. 2007;85:19-26.

25. Kelishadi R, Ardalan G, Gheiratmand R, Majdzadeh R, Delavari A, Heshmat R, et al. Smoking behavior and its influencing factors in a national-representative sample of Iranian adolescents: CASPIAN study. Preventive medicine. 2006;42(6):423-6.

26. AlBuhairan FS, Tamim H, Al Dubayee M, AlDhukair S, Al Shehri S, Tamimi W, et al. Time for an adolescent health surveillance system in Saudi Arabia: findings from "Jeeluna". Journal of Adolescent Health. 2015;57(3):263-9.

27. WHO. Physical activity [updated 26 November 2020]. Available from: https://www.who.int/en/newsroom/fact-sheets/detail/physical-activity.

28. Organization WH. Global action plan for the prevention and control of noncommunicable diseases 2013-2020: World Health Organization; 2013.

29. International W. Why is being physically active important? Available from: https://www.wcrf.org/policy/our-policy-work/.

30. Organization WH. Global action plan on physical activity 2018-2030: more active people for a healthier world: World Health Organization; 2019.

31. Sharara E, Akik C, Ghattas H, Obermeyer CM. Physical inactivity, gender and culture in Arab countries: a systematic assessment of the literature. BMC public health. 2018;18(1):1-19.

32. WHO. Tobacco Free initiative. Available from: http://www.emro.who.int/tfi/what-we-do/index.html.

33. Organization WH. Operationalization of the adolescent health component of the Global Strategy for Women's, Children's and Adolescents' Health, 2016-2030. World Health Organization. Regional Office for the Eastern Mediterranean, 2017.

\section{Tables}

Due to technical limitations, table 3 and 4 are only available as a download in the Supplemental Files section. 


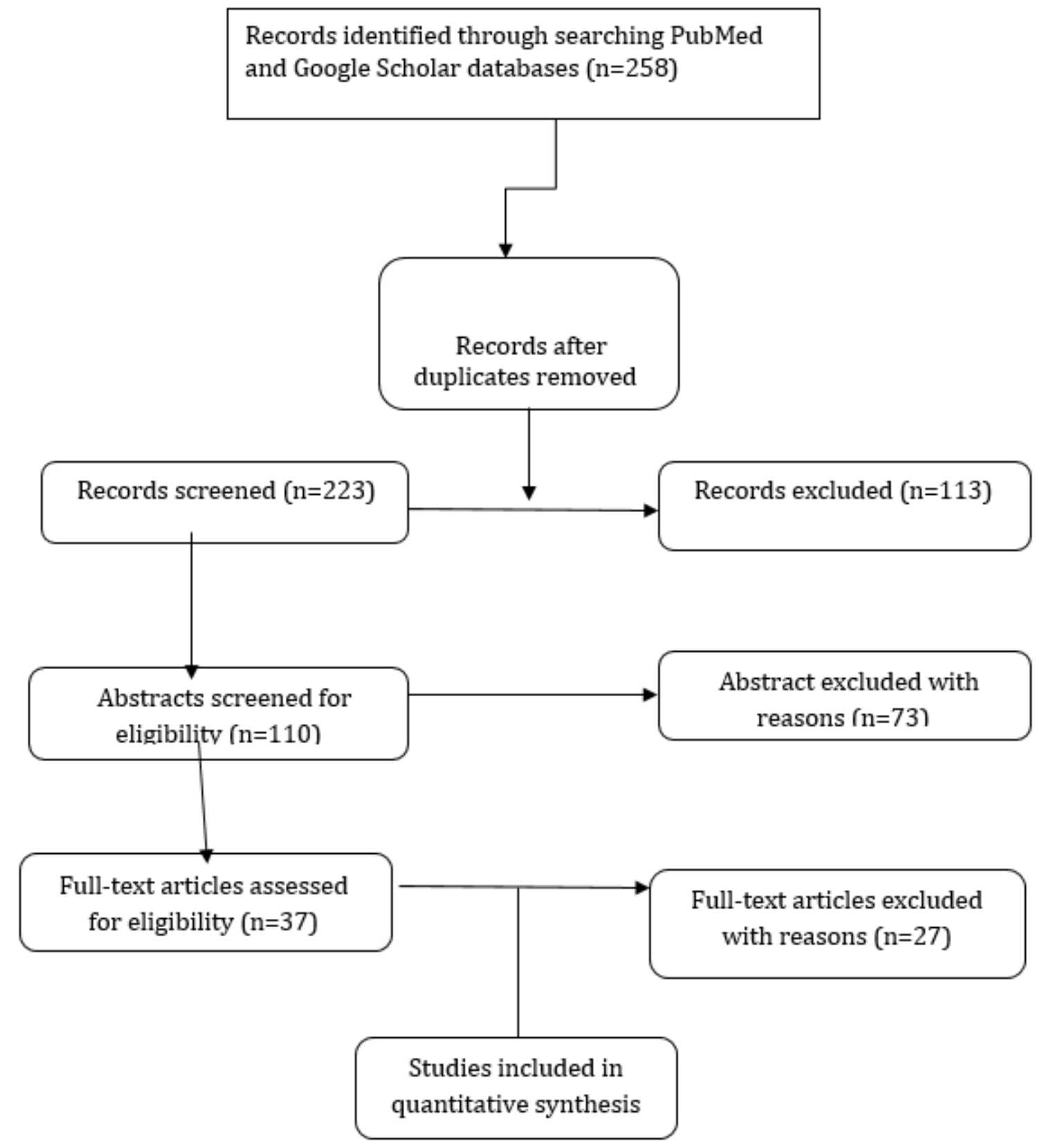

Figure 1

PRISMA Flowchart. The PRISMA flow diagram for the review detailing the database searches, the number of abstracts screened, and the full texts retrieved

\section{Supplementary Files}

This is a list of supplementary files associated with this preprint. Click to download.

- Appendix.docx 
- Table3and4.docx

Page 19/19 